\title{
High energy white beam $x$-ray diffraction studies of residual strains in engineering components
}

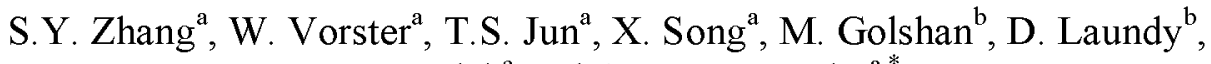 \\ M.J. Walsh ${ }^{\mathrm{c}}$ and A.M. Korsunsky, \\ ${ }^{a}$ Department of Engineering Science, University of Oxford, Parks Road, Oxford OX1 3PJ, UK \\ ${ }^{b}$ Synchrotron Radiation Source at Daresbury Laboratory, Warrington WA4 4AD, UK \\ ${ }^{c}$ Combustion Systems Engineering, Rolls-Royce plc, PO Box 31, Derby DE24 8BJ, UK \\ "Author for correspondence: alexander.korsunsky@eng.ox.ac.uk
}

\begin{abstract}
In order to predict the durability of engineering components and improve performance, it is mandatory to understand residual stresses. The last decade has witnessed a significant increase of residual stress evaluation using diffraction of penetrating radiation, such as neutrons or high energy X-rays. They provide a powerful non-destructive method for determining the level of residual stresses in engineering components through precise characterisation of interplanar crystal lattice spacing. The unique non-destructive nature of these measurement techniques is particularly beneficial in the context of engineering design, since it allows the evaluation of a variety of structural and deformational parameters inside real components without material removal, or at worst with minimal interference. However, while most real engineering components have complex shape and are often large in size, leading to measurement and interpretation difficulties, since experimental facilities usually have limited space for mounting the sample, limited sample travel range, limited loading capacity of the sample positioning system, etc. Consequently, samples often have to be sectioned, requiring appropriate corrections on measured data; or facilities must be improved. Our research group has contributed to the development of engineering applications of high-energy X-ray diffraction methods for residual stress evaluation, both at synchrotron sources and in the lab setting, including multiple detector setup, large engineering component manipulation and measurement at the UK Synchrotron Radiation Source (SRS Daresbury), and in our lab at Oxford. A nickel base superalloy combustion casing and a large MIG welded Al alloy plate were successfully studied.
\end{abstract}

Keywords: X-ray diffraction, residual strain, stress analysis

PACS: $61.05 . \mathrm{cp}$

\section{INTRODUCTION}

Reliable prediction of safe life of modern structural assemblies, such as automotive and aviation engines, requires detailed experimental characterization and theoretical description of their response to complex cyclic thermo-mechanical loading

CP1045, Current Themes in Engineering Science 2007, edited by A. M. Korsunsky (C) 2008 American Institute of Physics 978-0-7354-0573-8/08/\$23.00 
experienced in service. Large scale industrial, national and international research programs are carried out aimed at collecting the necessary experimental data, and developing suitably sophisticated modeling tools.

In recent years it has been established that residual stresses and prior deformation (e.g. during fabrication) experienced by structural components can exert a strong influence on the deformation behavior, and hence durability of engineering structural components. This study focuses particularly on the subject of evaluation of residual stress states, and the procedures for the incorporation of the relevant findings in predictive modeling methodologies for (thermo-mechanical) fatigue life prediction.

Recent experimental studies [1-8] have demonstrated that the use of high energy Xrays (up to and exceeding 100keV) allows diffraction experiments to be carried out in transmission through thick sections (exceeding several millimeters, and up to several millimeters) of important structural engineering alloys based on magnesium, aluminium, titanium, iron and nickel. Since these materials find extensive use in various parts of aerospace and automotive engine and body assemblies, reliable knowledge of residual stress states in manufactured components is essential for developing reliable and suitably precise predictions of thermo-mechanical fatigue resistance and durability under complex service loading conditions.

The principle of diffraction strain measurement in polycrystalline alloys relies on Bragg's law that establishes the relationship between the average interplanar lattice spacing $d$ within the sampling (or gauge) volume, on the one hand, and the wavelength (or energy) of X-ray photons and their scattering angle $2 \theta$, on the other:

$$
2 d \sin \theta=\lambda=h c / E,
$$

where $\lambda$ is the photon wavelength, $E$ is the photon energy, $h$ is Planck's constant, and $c$ is the speed of light. In practice the determination of interplanar spacing can be carried out in one of two distinct modes, monochromatic or energy dispersive. In the first case the wavelength (and energy) of the incident beam is defined to high precision (usually better than $\Delta E / E=10^{-3}$ ) by suitable optics, and the scattering angle $2 \theta$ is determined by scanning a point detector, or via the use of a position-sensitive detector (PSD). In the second case the scattering angle $2 \theta$ is maintained fixed, but the detector used possesses the capability to resolve the energy of photons arriving, and to count their numbers by placing each instance into appropriate bin with the help of pulse-height discrimination and multi-channel analyzer.

From the point of view of flux utilization and measurement efficiency, energy dispersive setup often turns out to be advantageous, since the total photon flux in the incident white beam (with wide energy spread) is vastly superior to monochromated beams. Furthermore, simultaneous collection of information about scattered intensity across a wide range of energies results in a diffraction pattern (that can be converted to intensity vs lattice spacing pattern using appropriate calibration, [8-11]) allows subsequent data interpretation that involves the refinement of a crystal lattice and scattering model (Rietveld refinement) through non-linear least squares fitting to the entire pattern containing multiple diffraction peaks. This allows the determination of 
average lattice parameters (e.g. $a, c$ ), and the corresponding lattice strains using the formula applicable to small strains:

$$
\varepsilon=\left(a-a_{0}\right) / a_{0} .
$$

Measurement arrangements and experimental studies described below demonstrate the capabilities of evaluating residual strains (and ultimately stresses) within large manufactured components using white beam high energy X-ray diffraction. In many cases the size and weight of real engineering components represents a challenge that can be addressed in one of several ways. On the one hand, suitably high capacity manipulation capabilities can be used to perform component scanning with respect to stationary beams. On the other hand, if a portable source can be chosen that provides radiation of sufficiently high flux and penetrating ability, then it is possible to make arrangements for the source (and the associated detection equipment) to be moved (scanned) with respect to large components, that in this case are maintained stationary. Finally, it is also possible to consider the possibility of performing controlled sectioning of large components into smaller parts in which residual strain measurements can be carried out, provided a suitable procedure can be identified for reconstruction of the residual stress state that existed in the entire component prior to sectioning.

Eigenstrain procedures [12-13] provide a general basis for residual stress reconstruction from a series of measurements of residual elastic strains, e.g. by diffraction; or measurement of strain increments that occur within the object during stepwise removal of material or cutting.

\section{EXPERIMENTAL SETUP AND DETECTOR ALIGNMENT}

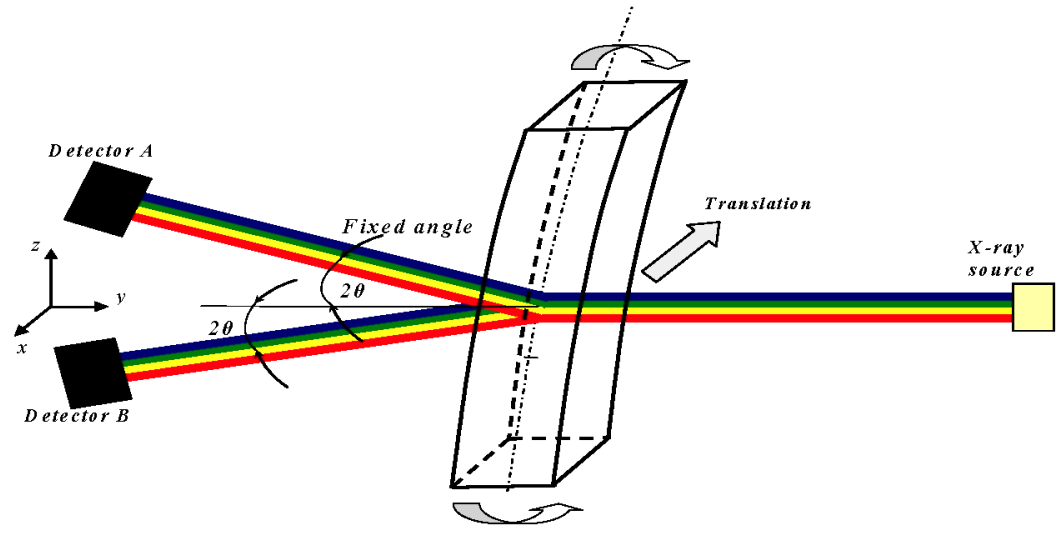

FIGURE 1. Schematic diagram of twin detector setup. Figure 1.

A laboratory high energy X-ray diffractometer for bulk residual strain evaluation (HEXameter) has been developed and built at Oxford University [14]. The principal 
difference between HEXameter and the synchrotron energy dispersive beamline is the nature of the X-ray source. Laboratory X-ray source was selected from industrial radiography range consisting of a high voltage $\mathrm{X}$-ray generator (up to $225 \mathrm{kV} / 4.5 \mathrm{~kW}$ ) equipped with a long-life metal-ceramic $\mathrm{W}$ target tube head.

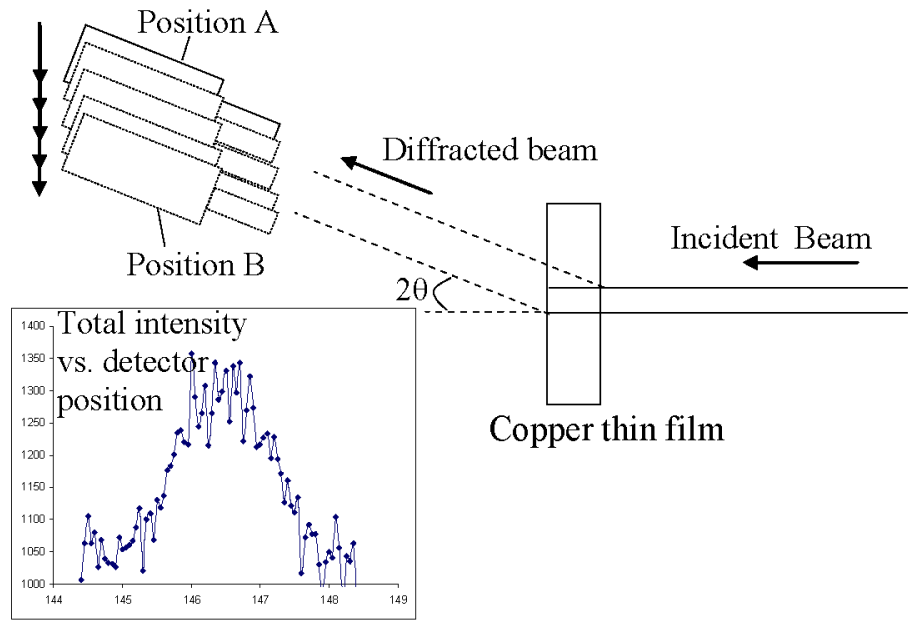

FIGURE 2. Schematic diagram of detector alignment procedure.

Synchrotron X-ray scattering experiments reported here were carried out at the Daresbury Synchrotron Radiation Source (SRS), on Station 16.3. The station is a high resolution facility utilizing the centre section of the 6 Tesla wiggler fan and producing a smooth white beam spectrum with useable photons having energies up to $100 \mathrm{KeV}$.

The principle of the experimental setup was the same for both laboratory diffractometer and Synchrotron beamline. The diffraction spectra were recorded using high-energy solid state detectors in HEXameter and SRS station 16.3. A multi-channel analyzer was used for data acquisition.

In most cases at least two mutually perpendicular directions of strains are needed for deduction of stresses. To increase data collection efficiency and obviate the requirement of rotating the sample, twin detectors setup was built in HEXameter and at SRS station 16.3. Fig 1 illustrates the principle of the twin-detector setup. Both detectors were positioned at small Bragg angles, but one was placed in the horizontal diffraction plane (spanning $x$ and $y$ axes), while the other was offset in the vertical plane (spanning $y$ and $z$ axes). The detectors collected counts at the same time, allowing simultaneous determination of strains in two mutually orthogonal directions that lie almost within the plane normal to the incident beam.

The challenge that arises in the use of twin-detector setup is correct alignment, that must ensure that the two detectors are collecting the data from the same (or almost the same) gauge volume position. The alignment procedure uses incident beam of small cross-section (e.g. $0.1 \mathrm{~mm} \times 0.1 \mathrm{~mm}$ ) to irradiate a thin film (less than $0.5 \mathrm{~mm}$ ) made from a fine-grained, strongly scattering material, such as copper. Provided adequate collimation is placed between each detector and the sample (detector slit and sample 
slit), strong diffraction signal will only be collected provided the collimators point at the small scattering volume.

We found it convenient to fix the scattering angle (e.g. by fixing it to be $10^{\circ}$ ) and scan the detector-collimation assembly perpendicular to the incident beam. Correct alignment of the detector corresponds to the position where the peak of total intensity is found. Fig. 2 illustrates the alignment procedure for the detectors.
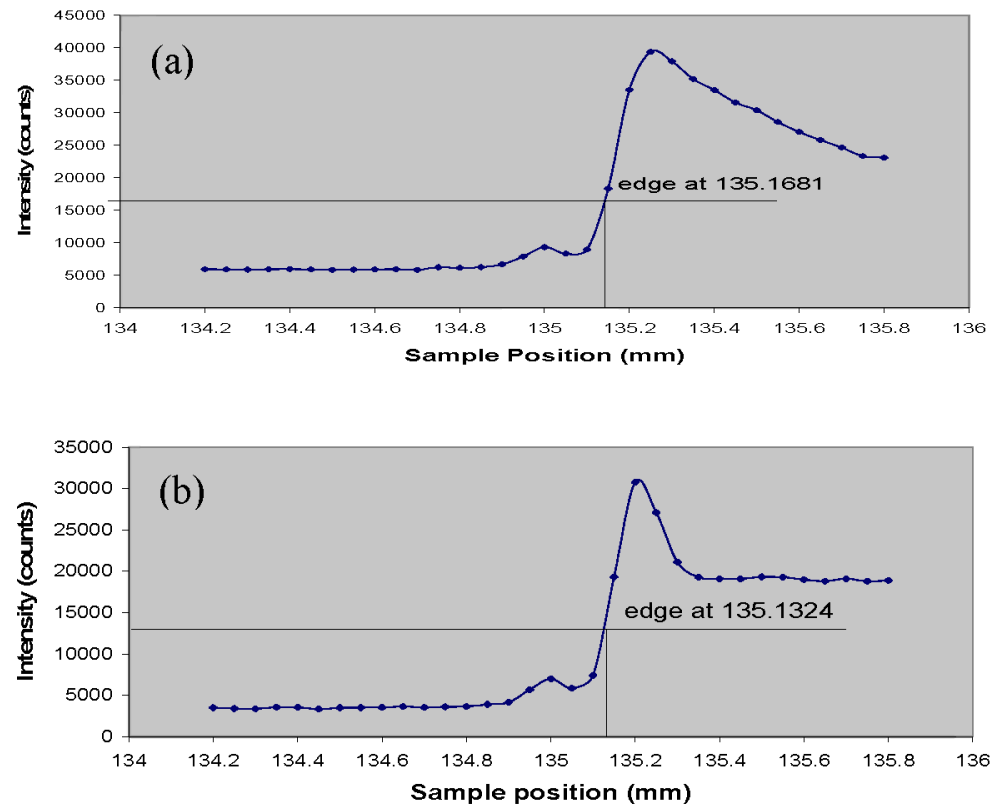

FIGURE 3. Schematic diagram of the surface finding procedure.

In order to check the accuracy of the alignment procedure, the two detectors were used to find the edge of an AlSiC bar of rectangular cross-section (illustrated in Fig.1). Figs. 3(a) and (b) show total intensity vs. sample position profiles for the vertical and horizontal detectors, respectively. The difference in the edge positions deduced amounted to less than 30 micrometres, suggesting that the accuracy of detector alignment that could be achieved using this approach was better than $1 / 3$ of the incident beam cross-sectional dimension.

The Oxford laboratory energy dispersive diffractometer HEXameter consists of the carrying frame, or yoke, that houses the source X-ray tube and associated collimation components, and the two energy dispersive detectors, with associated collimation components, as illustrated in Fig. 4. Co-alignment and calibration of the two detectors must be carried out prior to strain measurement, by following the alignment procedures described above, followed by collection of diffraction patterns from a powder sample with well-known crystal structure and lattice parameter, such as NIST silicon standard. This allows simultaneous determination of the precise scattering angle and of the channel-to-energy conversion characteristic for each detector. 
An important part of HEXameter is the high loading capacity (up to $100 \mathrm{~kg}$ ) translation-rotation system. to mount the component and a movable X-rays source. A motorized X-Y-Z- $\alpha$ positioning table was designed and made by MicroMech Systems Ltd, Braintree, UK. The translation stage is driven by micro-stepping motors and provides translation ranges of $500 \mathrm{~mm}$ and infinite rotation capability around the vertical axis.

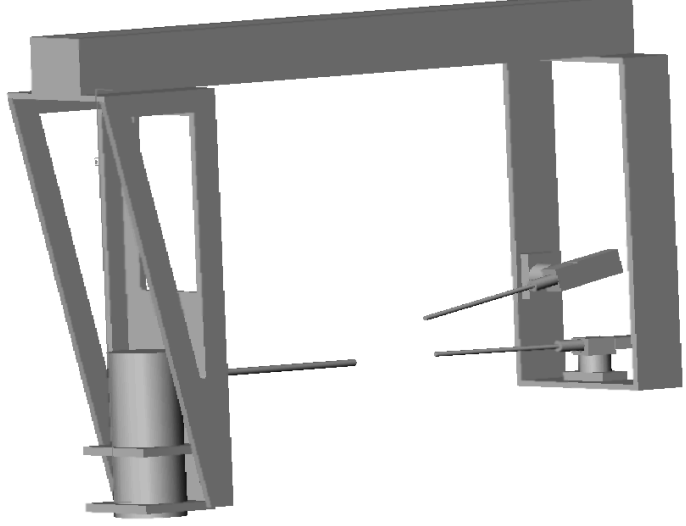

FIGURE 4. HEXameter 'yoke' with source and collimation 'nose' (left), and detectors (right).

Laboratory arrangement used in the HEXameter instrument provides an important aspect of manipulation flexibility, since the translation-rotation system can be used either to move the measured component with respect to the stationary 'yoke', or, alternatively, the 'yoke' can be attached to the translation-rotation system and moved with respect to the stationary sample. This setup flexibility will be used in the future to study large component, such as the combustion casing described below.

In contrast with the laboratory HEXameter setup, at SRS station 16.3 the only option available is that of moving the measured sample with respect to the stationary incident $X$-ray beam delivered from the insertion device. A high loading capacity translation system with $\mathrm{X}-\mathrm{Y}-\mathrm{Z}$-rotation sample positioning table was installed, and used to perform scanning operations described below.

\section{CASE STUDIES}

\section{Elasto-plastically bent $\mathrm{Al} / \mathrm{SiC} \mathrm{C}_{p}$ bar}

Diffraction measurements of elastic lattice strain were carried out using HEXameter and validated by comparison with the results obtained for the same samples on Station 16.3 at the SRS (Daresbury, UK). Fig. 5 illustrates the comparison between residual strain profiles within the alloy matrix of a bent $10 \times 10 \mathrm{~mm}^{2}$ cross-section bar of aluminium alloy matrix (AA2024) particulate (SiC) composite. Good quality of agreement between the two sets of measurements indicates that HEXameter provides an excellent tool for laboratory measurement of residual elastic strain in the bulk of specimens and engineering components. 


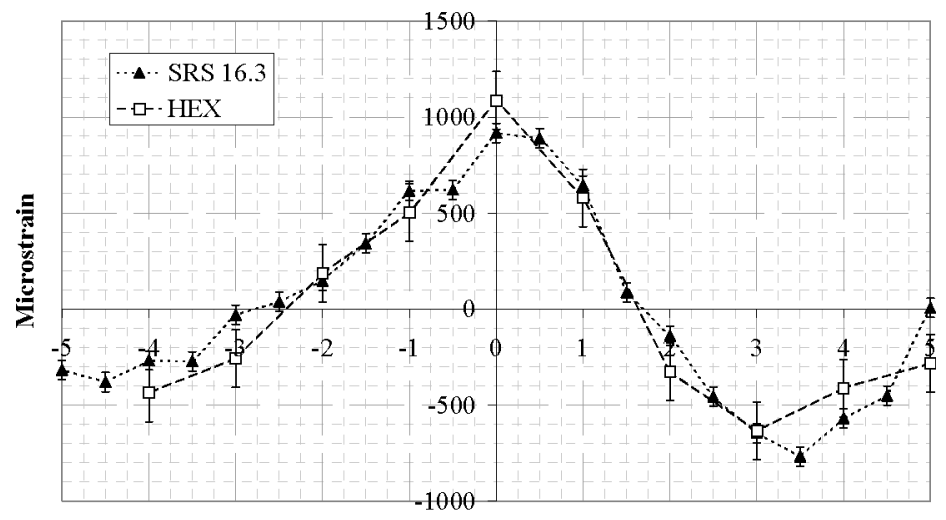

Position (mm)

FIGURE 5. Residual strain profile in the matrix phase of bent bar of Al alloy particulate composite.

\section{Aeroengine combustion casing}

Research project devoted to the analysis of structural integrity and durability of gas turbine engine combustion components (ATAP-10) was co-funded by the DTI and Rolls-Royce plc. It focused principally on combustion liners and casings, aiming to produce a validated lifing methodology for the design and in-service support of a wide variety of gas turbine components. The casing has the dimensions of $880 \mathrm{~mm}$ (maximum diameter) by $750 \mathrm{~mm}$ (height), a mean wall thickness of about $3 \mathrm{~mm}$, weighs over $50 \mathrm{~kg}$. It has complex geometry allowing for the mounting of fuel supplies and liners, and has been manufactured by using forging followed by machining, as well as electron beam welding. Combustion casings have circumferential flanges at their rear and front ends for the purposes of providing bolted connection to other engine components downstream and upstream from the combustor.

The material of combustion casing is nickel base superalloy IN718. Like many superalloys, this material retains strength to temperatures that constitute an unusually high proportion of its melting temperature. Its strength is derived from the distribution of precipitates of $\gamma^{\prime}$ phase that provide effective obstacles to dislocation movement and thus increase the yield stress of the material and improve its resistance to high temperature creep deformation.

The residual stress state within the casing is complex, and would be disturbed if the casing were to be sectioned to extract coupons of conveniently small dimensions. Due to the size and shape of the combustion casing considered here, experimental set up at Station 16.3 (SRS Daresbury, UK) required the use of large capacity manipulation stage described earlier. In particular, the casing was tilted on the table to a predefined angle in order to ensure that a carefully defined component of residual elastic strain was measured, and that the diffracted beam was not blocked from reaching the detector. The setup is illustrated in Fig. 6. Note that twin detector set up was used that was similar to that shown in Fig. 1 for laboratory measurement using HEXameter diffractometer. 


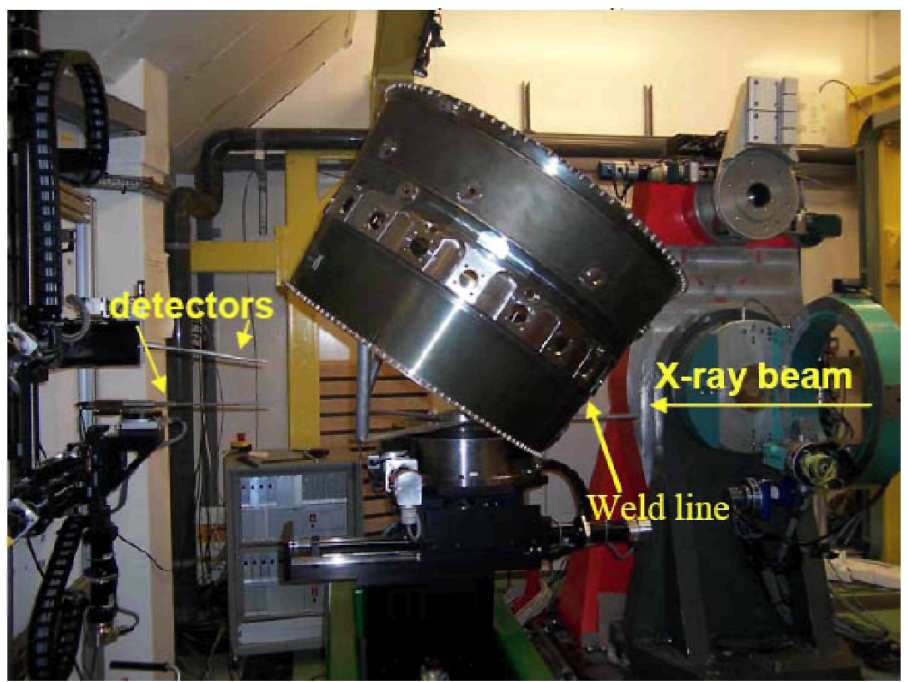

FIGURE 6. Illustration of the setup of combustion casing on the large capacity manipulator at Station 16.3, SRS.

In our analysis we were particularly concerned with certain locations within the casing where the combination of geometric characteristics and the loading conditions experienced in service made the initiation of fatigue crack most likely. Fig. 7 provides a more detailed indication of the location within the casing at which a line profile of average macroscopic residual elastic strain was collected. The scan line extended from the round boss towards the circumferential weld joint line.

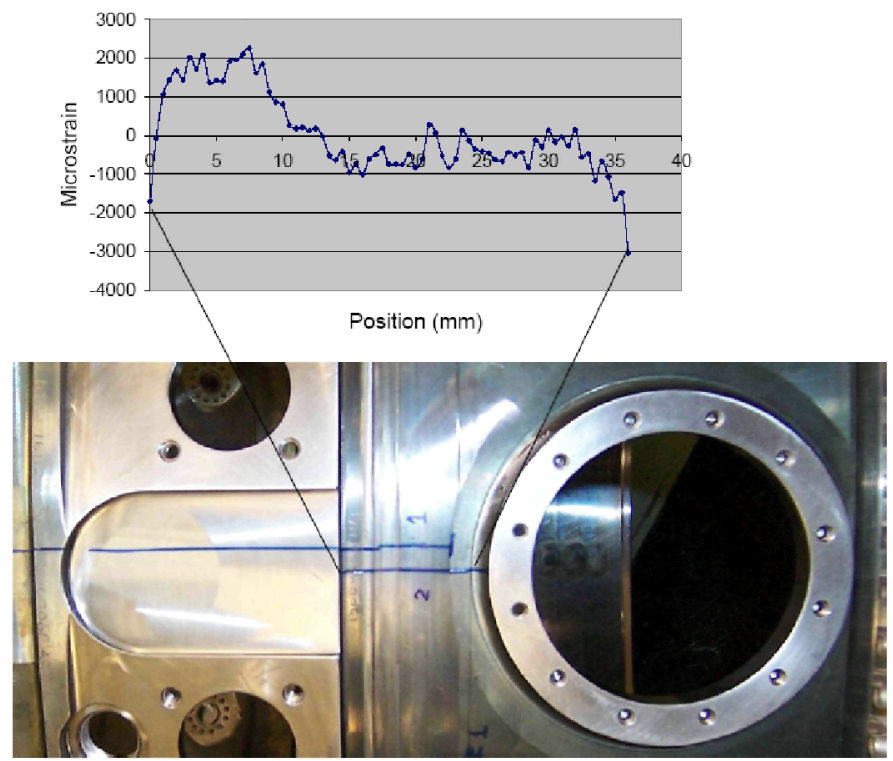

FIGURE 7. Detail of the combustion casing and the scan line (marked 2) and the corresponding measured residual elastic strain profile. 
The hoop component of the residual elastic strain indicated in the graph shown in Fig. 7 shows the tensile region associated with the weld that is centered around the position $5 \mathrm{~mm}$. This tensile hoop residual elastic strain decreases to low (near zero) levels at distances of about $5 \mathrm{~mm}$ each side of the weld center line, becoming slightly compressive towards the round boss seen in Fig. 7. Precise determination of the magnitudes of two significant stress components (hoop and axial) requires the collection of multiple strain components within the casing. Once such data is obtained, stress determination can be carried by a variety of techniques, e.g. the $\sin ^{2} \psi$ method.

\section{CONCLUSIONS}

The significance of the work described in the present study lies in the possibility of using the developments reported here to derive improved fatigue lifing procedures that must incorporate the influence of residual stresses on deformation behaviour, crack initiation and crack propagation under in-service conditions.

Large capacity manipulating equipment has become a standard requirement for dedicated engineering instruments at large research facilities, such as the engineering diffraction instrument ENGIN-X at the ISIS spallation neutron source at the Rutherford Appleton laboratory near Oxford, UK. Diamond, the new UK synchrotron light source that is located on the same site, will house JEEP, Joint Engineering, Environment and Processing beamline for monochromatic and energy dispersive diffraction and imaging. JEEP will have capabilities for precise positioning of large components and loading devices weighing up to $500 \mathrm{~kg}$, combined with advanced detectors, such as the multi-cell energy dispersive detector for simultaneous data collection for up to 23 different scattering vectors. This will open up prospects of efficient acquisition of large data sets used to refine and validate sophisticated numerical models of residual stress states within components of complex geometry.

The influence of residual stress (or internal hardening state) on fatigue resistance of materials represents a separate area of research not dwelt upon in the present study. Instead, in the present context the principal interest was placed on the ability to determine the residual stress distribution in greatest level of detail possible, and subsequently to incorporate this information in numerical models of entire components or assemblies. This begins with experimental determination of residual strain state, in the present analysis accomplished by diffraction of high energy X-rays.

Residual elastic strains may only ever be determined as average values within certain gauge volumes that are sampled at a certain finite number of measurement

positions. Reconstruction of the complete residual stress state from this finite set of measured values, and incorporating this information within the finite element model of the component is the next task. The eigenstrain modeling approach seems to provide an efficient and convenient method of representing the sources of residual stress, allowing subsequent in-service deformation to be considered, and residual stress evolution to be considered.

Future development of residual stress analysis methods is likely to be progressively 
more closely linked with deformation modeling. It is also likely that the range of scales accessible to residual stress measurement techniques will expand, both towards larger objects (such as buildings, bridges, and other civil engineering structures) and towards smaller length scales (electronics components, micro- and nanoelectromechanical systems, etc.) While measurement techniques suitable to these vastly different scales must necessarily be different, some of the interpretation and analysis techniques, such as eigenstrain reconstruction procedures for residual stress states, possess sufficient generality to be useful across length scales, from macroscopic to micro- and nano- scales [16].

\section{REFERENCES}

1. A.M. Korsunsky, S.P. Collins, R.A. Owen, M.R. Daymond, S. Achtioui, K.E. James (2002) Fast residual stress mapping using energy-dispersive synchrotron $X$-ray diffraction on station 16.3 at the SRS. J Synchrotron Radiat, 9, p.77-81.

2. A.M. Korsunsky, W.J.J. Vorster, S.Y. Zhang, D. Dini, D. Latham, M. Golshan, J. Liu, Kyriakoglu Y., Walsh M.J. (2006) The Principle of Strain Reconstruction Tomography: Determination of Quench Strain Distribution from Diffraction Measurements, Acta Materialia 54 (8), p. 2101-2108.

3. A.M. Korsunsky, J. Liu, M.Golshan, D. Dini, S.Y.Zhang, W.J. Vorster (2006) Measurement of Residual Elastic Strains in a Titanium Alloy Using High Energy Synchrotron X-Ray Diffraction, Experimental Mechanics, 46 (4), p. 519-529.

4. W. J.J. Vorster, S. Y. Zhang, M. Golshan, D. Laundy, D. Dini and A. M. Korsunsky (2007) Comparison of X-ray diffraction measurement of residual elastic strains using monochromatic image plate data and white beam energy-dispersive analysis, J. Strain Analysis, v.42 (1), p. 23-37.

5. M. Topic, R. Bucher, W. Vorster, S.Y. Zhang, P. McGrath, A.M. Korsunsky, Residual stress in laser bent steel components, Materials Science Forum (2006) 524-525, p. 299-304.

6. A.M. Korsunsky, M.R. Daymond, K.E. James (2002) The correlation between plastic strain and anisotropy strain in aluminium alloy polycrystals. Mater Sci Eng A - Struct Mater Prop Microstruct Process, 334, p.41-48.

7. A.M. Korsunsky, K.E. Jarnes, M.R. Daymond (2004) Intergranular stresses in polycrystalline fatigue: diffraction measurement and self-consistent modeling. Eng Fract Mech 71, p.805-812.

8. J. Liu, K. Kim, M. Golshan, D. Laundy, and A.M. Korsunsky (2005) Energy calibration and fullpattern refinement for strain analysis using energy-dispersive and monochromatic X-ray diffraction. J. Appl. Crystallography, 38, p.661-667.

9. P. Ballirano, R. Caminiti (2001) Rietveld refinements on laboratory energy dispersive X-ray diffraction (EDXD) data. J Appl Crystallogr 34, p.757-762.

10. A. Steuwer, J.R. Santisteban, M. Turski, P.J. Withers, T. Buslaps (2004) High-resolution strain mapping in bulk samples using full-profile analysis of energy-dispersive synchrotron X-ray diffraction data. J Appl Crystallogr, 37, p.883-889.

11. A.C. Larson, R.B. Von Dreele (2000) General Structure Analysis System (GSAS) manual.

12. A.M. Korsunsky (2005) On the modelling of residual stresses due to surface peening using eigenstrain distributions. J Strain Anal Eng Des, 40, p.817-824.

13. A.M. Korsunsky (2006) Variational eigenstrain analysis of synchrotron diffraction measurements of residual elastic strain in a bent titanium alloy bar. J Mech Mater Struct, 1(2), p.259-278.

14. A.M. Korsunsky, S.Y. Zhang, D. Dini, W.J.J. Vorster, J. Liu (2006) Oxford HEXameter: Laboratory high energy X-ray diffractometer for bulk residual stress analysis, Materials Science Forum, 524-525, p. 743-748.

15. Y. Zhang, M.E. Fitzpatrick, L. Edwards (2005) Analysis of the residual stress around a coldexpanded fastener hole in a finite plate, Strain, 41 (2), p.59-70.

16. A.M. Korsunsky, S. Cherian, R. Raiteri, R. Berger (2007) On the micromechanics of microcantilever sensors: property analysis and eigenstrain modeling, Sensors and Actuators A Physical, 139, p. 70-77. 